\title{
Collaborative Systems for Smart Environments: Trends and Challenges
}

\author{
Luis M. Camarinha-Matos ${ }^{1}$ and Hamideh Afsarmanesh ${ }^{2}$ \\ ${ }^{1}$ Faculty of Sciences and Technology, Universidade Nova de Lisboa / Uninova, Portugal \\ cam@uninova.pt \\ ${ }^{2}$ Informatics Institute, University of Amsterdam, The Netherlands \\ h.afsarmanesh@uva.nl
}

\begin{abstract}
Collaborative systems will form the warp for smart networked environments wherein humans, organizations, intelligent agents, and devices collaborate. The smart environments of near future will be context sensitive systems within which the physical world is richly and transparently interwoven with sensors, actuators, and computational elements that seamlessly embed everyday objects and interconnect them through networks. Modeling, design, and development of collaborative systems in this context will support a large number of emerging applications including security, care and assistance, transportation, construction, sustainability and energy management, education, government, and manufacturing. In this context, a brief survey of trends and challenges is presented.
\end{abstract}

Keywords: Smart Environments, Collaborative Networks, Cyber-Physical Systems.

\section{$1 \quad$ Introduction}

Smart environments represent a fast growing area which is often presented as an evolution of the notion of pervasive or ubiquitous computing, initially suggested by Mark Weiser [1] as "a physical world that is richly and invisibly interwoven with sensors, actuators, displays, and computational elements, embedded seamlessly in the everyday objects of our lives, and connected through a continuous network". From this notion, smart environments emerge as "small worlds that are able to acquire and apply knowledge about the environment and its inhabitants in order to improve their experience in that environment" [2]. Similar to pervasive computing, in smart environments there is the idea of a physical environment interwoven with a network of devices and systems, sensors and actuators. But now with a more explicit purpose using technology to improve the life or comfort of its inhabitants.

Examples of smart environments include: closed spaces with relatively welldefined boundaries, such as home, office, or car, and open spaces such as streets, bridges, parking lots, or smart cities [3]. The relevance of this area can be illustrated by the growing number of applications in domains such as health monitoring, elderly care provision, transportation and logistics, entertainment, environment monitoring, smart homes, smart offices, and smart shop floors, etc. 
In technical terms, smart environments result from the convergence of a variety of contributions including sensor networks, pervasive and mobile computing, robotics, miniaturization of hardware components with embedded processing power, reliable wireless network protocols, increased automation associated to everyday devices such as home appliances, natural human-computer interfaces, artificial intelligence, middleware platforms, etc. In fact, this is clearly an area that is leveraged by multidisciplinary contributions.

When systems involved in smart environments grow and involve large number of entities (hundreds, thousands, or millions), flat organizational structures are not sufficient to support effective management. Therefore we also need to address the organization of such entities in "communities" and "societies" within the "ecosystems" of cyber-physical artefacts. Within such organizational structures collaboration among artefacts can then support the creation and provision of composed value-added services, by service developers. Such environments and associated services are opening new grounds for business opportunities, which typically require the collaboration of multiple stakeholders, and also challenge the positioning of enterprises in society, leading to new concepts such as sensing enterprise. Collective awareness then also becomes a necessary property of such systems. Furthermore, some artefacts have a nomadic characteristic, i.e. they move between spaces (e.g. gadgets or sensors carried by the human user or by other moving objects), which in turn raises the issues of artefact discovery, acceptance (by the "visited" ecosystem), definition of roles, access policies (addressing security and safety), pro-active promotion of services, and system dynamics. In this context, data/service interoperability assumes a fundamental role.

Additionally, people are increasingly surrounded by and depend on a fast growing collection of gadgets and other smart objects in their daily lives. These artefacts in turn have gradually changed people's habitat and extended their sensorial and acting capabilities. User acceptability is therefore the base criterion for the design of any such systems, in the context in which users' expectations increase as the technology evolves. Another important criterion for designing such systems is the trustworthiness of the components and the composed systems, which is mainly associated to the notions of reliability, risk, privacy, and security. Furthermore, with the trend towards more personal data being available through the cloud, monitoring and understanding "what people will accept" in exchange of higher value services, becomes relevant. On the other hand, smarter decision making and higher levels of autonomy is required from systems, in order to neither "overload" the humans with excess of information, nor requiring constant input, e.g. "pressing buttons" to interact with the cyber-physical world, considering the real-time access to a large number of devices.

In this context, collaborative networks can play a significant role in a number of areas facilitating the design and development of complex smart environments. Although already identified by various authors [2], [3], such collaborative perspective is so far only briefly touched by current developments.

This introductory text aims at giving a brief overview of the area and its challenges, particularly from the collaborative networks perspective, contributing to identify further research directions. 


\section{Related Areas}

A number of different partially overlapping related paradigms have been introduced in recent years, among which the Ambient Intelligence (AmI), Ambient Assisted Living (AAL), and Sensing Enterprises can be named. These paradigms address environments supported through the collaboration among human actors, intelligent agents, and smart devices.

Different aspects of the above paradigms are further addressed by related work in several technology-based areas, including the Cyber Physical Systems (CPS), Internet of Things (IoT), Internet of Events, Sensing Networks, etc.

As an outcome of these research and development directions, a number of advanced areas of application have emerged illustrating substantial impact in improving the quality of life in societies, as exemplified by the Smart Cities, Smart Homes, and a number of Intelligent Infrastructures such as those manifested as Intelligent Transportation Systems and Smart Grid, etc.

\subsection{Related Paradigms}

Ambient Intelligence (AmI): A paradigm that represents electronic-enhanced environments, which are sensitive and responsive to the presence of people [4], considering their needs, habits, and even emotions. The notion is similar to smart environment, but perhaps with more emphasis on human-computer interaction, context awareness, and provision of intelligent services.

A number of characteristics are usually presented to identify AmI systems, including: context awareness, personalization (tailored to the needs of each individual), acting anticipatory (anticipating the needs of individuals), adaptive (coping with the changing needs of individuals), ubiquitous (integrated in our everyday environments), and transparent (making computers disappear in the background) [4], [5].

Many application examples can be found in the areas of healthcare and elderly care [5], but references in other sectors can also be found [4], [6], e.g. in smart homes, intelligent transportation, rescue / crisis management, education, workplaces, energy management, etc. The convergence between AmI and social computing is attracting growing interest [7], namely with the new interaction facilities offered by mobile computing and wireless networks.

Ambient Assisted Living (AAL): AAL is a paradigm widely used in Europe representing a kind of AmI that provides safe and adapted environments for people with specific needs, allowing them to live more independently [8]. Most developments in AAL are focused on assisting elderly [9], [10], namely to help them live independently in their home environments. Most efforts in the last decade have been focused on transferring the dependence from human assistance to assistive devices [11]. But even earlier works such as the TeleCARE project [12], [13], pointed out the need for a collaborative systems approach to AAL, both at the infrastructure 
level (collaborating multi-agent systems) and social computing level (virtual communities).

In fact, the realization that a pure technological perspective also has the negative effect of reducing the social connections of the assisted people has triggered other efforts to combine the basic technological assistive aspects with social computing [10]. Furthermore, provision of integrated care and assistance services require the involvement and collaboration of multiple stakeholders. This led to a shift in the AAL developments, from a device and infrastructure focus, to a care ecosystem [14], [15]. Such perspective is well represented in the BRAID roadmap for ICT and ageing [16] and recent development initiatives such as the AAL4ALL project [9], [10].

Sensing enterprise: A paradigm introduced by the FInES (Future Internet Enterprise Systems) cluster of projects [17] to refer to an enterprise making decisions by using multi-dimensional information captured through physical and virtual objects in its environment, and providing added value information to enhance its global context awareness. In other words, this notion particularly focuses on enriching enterprises' context awareness through intelligent, interconnected, and interoperable smart components and devices that power enterprise systems, making them responsive to events in real time and aiming at reaching seamless transformation of (raw) data to (tailored) information and (experienced) knowledge [18].

\subsection{Related Supporting Technologies}

Cyber-Physical Systems (CPS): Engineered systems that are built from and depend upon the synergy of computational and physical components [19]. Numerous projects (e.g. AMADEOS, CASAGRAS2, CONET, GAMBAS, SCUBA, COMPASS, DANSE, DYMASOS, to name a few), have addressed the development of communication middleware systems to support seamless and trustworthy interoperation of heterogeneous subsystems and artefacts, new programming paradigms to support adaptive applications, methods to deal with real-time requirements, and management of devices with critical constraints such as energy, computing and communication capabilities. Many successful developments have been focused on vertical use cases, while those solutions prove to be difficult to generalize and scale up. The cost-effective engineering of larger CPSs, coping with an evolving nature and ensuring added-value to their users, remains a challenge.

Internet of Things (IoT): A term representing "a dynamic global network infrastructure with self-configuring capabilities based on standard and interoperable communication protocols where physical and virtual "things" have identities, physical attributes, and virtual personalities, and use intelligent interfaces, and are seamlessly integrated into the information network" [20]. In this context, a "thing" is understood either as a real/physical or digital/virtual entity that exists and moves in space and time and is capable of being identified [21]. Large classes of the objects that are connectable to Internet constitute (smart) sensors and (smart) devices, able to provide status information, thus an important technological enabler for smart 
environments. IoT is in fact a way to materialize the vision of a technology that becomes invisibly embedded in our natural surroundings, but present themselves whenever we need them.

There has been quite some discussion on the relationship between Cyber-Physical Systems and Internet of Things. For some authors the two terms carry some "geographical bias", being CPS more popular in the USA, while IoT is more prevalent in Europe and Japan. Others refer to CPS as being originated in the embedded systems community, while IoT comes from the area of Internet computing. These are certainly limited perspectives, and other authors try to relate the two concepts in terms of their focus [21], [22]. In any case, there is a growing convergence between the two areas since CPSs are becoming more Internet-based and complex CPSs / complex Systems-of-Systems increasingly combine open systems with closed and semi-closed ecosystems.

Internet of Events: Although it is less popular than IoT, it corresponds to a perspective of the IoT that puts the emphasis on time dependency and discrete events handling [41]. As such, events modeling and management, time critical reactivity, and process modeling and supervision are the relevant issues here.

Sensor Networks: Being implicit in all the above areas, sensor networks are a fundamental component of any smart environment which relies first and foremost on sensory data acquired from multiple sensors in distributed locations of physical environments. One of the most relevant topics in this area is the wireless sensor networks which typically comprise large numbers of spatially distributed autonomous sensors that have limited computing, communication, and energy autonomy capabilities. In this context, collaboration appears as a way to cope with those limitations, leading to the emergence of the notion of collaborative sensor networks. Examples of collaboration in such networks include [23]: collaboration in localization, energy awareness and collaboration to reduce energy consumption and extend system's lifetime, collaborative sensing and perception, collaboration in sending messages through different paths in response to security threats, self-adapting routing, covering "sensing holes" resulting from inactive nodes, etc.

Closely related to the increase in the sensing capabilities, the term big data has become popular. It refers to massive data sets and stream computing that due to their large size and complexity are beyond the capabilities of traditional databases and software techniques. The expansion of sensing capabilities, sensor networks, smart devices, and other sources, generating huge amounts of data, motivates the importance of this topic. Interest in big data has emerged in science, business, security, and many other areas [17]. Context awareness or collective awareness, supporting intelligent services for smart environments, may require the adoption of techniques being developed for big data.

\subsection{Advanced Application Areas}

Smart Home: Sometimes also known as home automation, are smart environments at home that rely on networked technologies to provide inhabitants with better comfort 
and security [24], [25]. Typical functions include monitoring and control of lighting, temperature and ventilation, home access security, entertainment, control of home appliances, etc.

Although home automation technologies have been around for the last thirty years, for a while, their developments evolved in isolated niches. Recent efforts are focusing on integrated solutions and the possibility of integrating different artefacts in a technology-agnostic manner, as aimed by initiatives such as openHAB [26], FHEM [27], or openRemote [28]. In recent years there has been a growing attention devoted to energy awareness systems and energy efficiency, an opportunity to integrate smart home and smart grid technologies [29]. Developments in Internet of Things, natural user interfaces, wireless sensor networks, and service robotics are also adding new dimensions to this area [30], [31].

Smart Cities: The concept of smart city has been gaining increasing importance in the research and policy making agendas, with many initiatives being promoted worldwide. And yet, the concept is not precisely defined, corresponding to different visions for different communities and stakeholders, also with variants according to geographical origin. A high level notion is that a smart city must be "able to optimise the use and exploitation of both tangible (e.g. transport infrastructures, energy distribution networks, natural resources) and intangible assets (e.g. human capital, intellectual capital of companies, and organisational capital in public administration bodies)" [32]. The results of an operational definition in this direction is presented in [33] that was applied to evaluate the degree of smartness of 70 medium-sized European cities, includes six dimensions of analysis: (i) "smart mobility", (ii) "smart environment", (iii) "smart governance" (iv) "smart economy", (v) "smart people", (vi) "smart living".

From an ICT focus, smart cities are characterized by pervasive computing and extensive sensing and information integration capabilities, in the various urban sectors, to help cities make better use of their resources. Implicit in all pilot initiatives towards building smart cities, is the need to engage and promote collaboration among multiple stakeholders, both public institutions and private organizations, as well as citizens. As such, collaborative networks can bring a fundamental contribution to this domain. As many projects are still at the level of building the base infrastructures, collaborative networks are not yet explicitly visible but undoubtedly they are a must when progressing towards providing integrated services.

Intelligent Infrastructures: are infrastructures that use computing and communication technologies, sensors, and other networked devices, to deliver relevant real-time data to their operators in order to optimize their use, minimize costs and prevent failures [34], as well as proving higher-level services to their users.

One example is given by the Intelligent Transport Systems, which are leading to the progressive introduction of new technologies based on electronics, sensorial systems, and communication and information technologies. For instance, road management policies, based on user-paying models, and the increasing concerns about traffic safety, establish requirements for a new family of emergent business 
services. A promising direction aims at offering new comprehensive service contracts integrating multiple possibilities of access to public transport systems, parking areas, subscription to innovative insurance policies, etc. [35], [36]. Another challenge is the development of collaborative eco-driving environments, focused on effective support of integrated services targeting transportation energy efficiency, costs saving, and improvement of safety in mobility.

Another example is the Smart Grid, or smart energy grid, which represents a move from a centralized, energy producer-controlled network to one less centralized and more consumer-interactive. Initially, it corresponds to an overlay of the energy distribution grid with an information and (smart) metering system [37], [38]. In the current stage, most efforts are very focused on infrastructure aspects. However, establishing a truly smart grid requires the participation of a large number of stakeholders, including producers, transmission and distribution operators, regulators, policymakers, and consumers. Thus, a next challenge is to adopt proper organizational models, governance structures, and to develop advanced tools to support collaboration among these players [39].

\section{Some Technical Challenges}

Earlier developments of smart environments, often led by specific application needs, contributed to the identification of key technical challenges and preliminary evaluation of promising solutions, some of which were briefly mentioned above. The urge to develop more advanced environments for diversified application domains raises various critical challenges, including:

\subsection{Modeling Approaches and Collaboration Needs}

Modeling is a fundamental part of the development of future smart environments. Although various modeling approaches have been used, a number of aspects need to be further addressed, including:

- How to represent objects / devices in the cyberspace. Two main approaches have been used: agents and service orientation. The first one is particularly appealing when modeling objects / subsystems with some level of autonomy and reasoning capabilities. In practical terms, this approach can be well supported by existing multi-agent platforms when focusing local, well-confined environments. When addressing larger physical environments, agent platforms have some limitations. Modeling devices through the services they provide is another common approach, which is not constrained by geographical limits, but does not properly encapsulate all relevant aspects of the object being modelled. Efforts to combine the two paradigms, as exemplified by the service entity notion [42], can be a good direction. Furthermore, when dealing with physical systems, it is also relevant to model events and pro-activity of devices / sub-systems [43].

- How to discover the objects / devices and their functionalities. Independently of the modeling approach, it is necessary to provide registry and discovery 
mechanisms that also cope with the nomadic and volatile nature of some devices. For instance, devices / entities can "appear and disappear" from the cyber-space, or evolve over time. Associated to this aspect, it is necessary to also model access rights, which are closely related to the ownership of devices / subsystems and the business models available within the smart environment.

- Organizational structures. Besides the individual components, it is also necessary to model the organizational structures present in the environment. Particularly, it is necessary to model the various categories of networked entities and their organizational structures, e.g. devices, sensors, machines, enterprises/organizations, and human actors, covering both their functional and behavioral characteristics, as well as their specificities, e.g. mobility and evolution perspectives in this environment.

- Business services modeling. In a complex smart environment, some of the services offered to its inhabitants are likely to be jointly provided by various stakeholders.

Dealing with the complexity arising from the large and increasing number of artefacts and subsystems constituting smart environments, and the inherent dynamism involved in such environments, suggest organizing them around different "spaces", constituting certain "communities" or "ecosystems" and "societies" of artefacts. Within such organizational structures collaboration among artefacts and subsystems is a requirement for the creation and provision of composed value-added services for the benefit of the environment inhabitants. To support value-added service creation, mechanisms are required for registering and sharing service components within service-developers communities, as well as supporting these developers with discovery and integration of such component services.

As such, collaborative networks concepts need to be applied to various levels, including: (i) giving an organizational scope to the structure of the smart environment and supporting collaboration among artefacts and subsystems, (ii) supporting the multiple stakeholders that participate in the building and operation of the smart environment, (iii) providing a framework for interaction between users and environment services, and (iv) coping with the communities of users and their organizational structures.

\subsection{Other Technical Aspects}

Human-systems interaction. In order to effectively improve life/comfort of inhabitants of the smart environments, new and advanced forms of interaction with users need to be considered. For instance, natural user interfaces and augmented reality, benefiting from well-proven techniques (e.g. from the gaming industry) are likely to play a relevant role here. In this area, it is also necessary to further handle a number of other aspects, such as: dealing with the "excess of information" that comes from large variety of sources, reducing the effort of users having to deal with many different interfaces, capturing user experience to adjust the systems to the user's life style and preferences, etc. 
Technological artefacts in smart environments are gradually changing people's habitat, while extending people's sensorial and acting capabilities in these environments. Therefore, the challenge is not only to consider the human in the loop when designing systems, rather the fact that the systems shall be human-centric. On the other hand, users can have a growing influence on the smart environments innovation process, namely through social networks, forums, blogs, rating systems, and similar mechanisms, which raises the importance of the concept of co-innovation in this area.

Risks and security. As the environments become increasingly permeated by technology and networked infrastructures, and their inhabitants become more dependent on such technology, the issue of trustworthiness of the components and systems, which is associated to the notions of reliability, risk, privacy, and security, assumes critical relevance. Therefore, the definition of environment-level trustworthiness indicators and their monitoring mechanisms, combined with recovery procedures contributing to system's resilience, need to be developed.

Furthermore, when smart environments are addressed as societies of artefacts and sub-systems, it is important to study and manage their emergent behaviors. Some behavioral patterns are "healthy" in the sense that they are aligned with the environment's purpose, generating value to the human inhabitants, while others are "faulty", due to either malfunctioning or cyber-attacks. Systems resilience is then a desired property, calling for new indicators of "system's health" and associated monitoring and diagnosis functionalities.

Technological basis. Smart environments involve a large diversity and increasing quantity of devices and infrastructures with different life cycles. In this context, interoperability and scalability are rather critical issues. The nomadic nature of many devices / gadgets that may enter / leave the environment also poses strong interoperability requirements. Therefore, the ability to easily integrate different artefacts in a technology agnostic manner remains a fundamental goal [26], [27], [28].

Technological developments need to also deal with the peculiarities of devices regarding their constraints on energy, communication range, computational power, memory capacity, etc., for which collaborative approaches can also provide ways to overcome individual limitations. Early examples in this direction can be found in the approaches to implement energy aware collaborative sensor networks [23].

Bridges to other fields. The growing availability of cloud computing resources can provide a solution to the constraints of some devices in widely distributed smart environments and thus a close link between the two areas needs to be explored.

Being smart environments potential sources of large volumes of data, a close link with the developments in big data / data science may open opportunities for the creation of new advanced business services.

\subsection{Engineering Methods}

While current engineering techniques have been good enough to deliver small-scale smart environments, they appear rather limited when addressing complex systems 
requiring a convergence of different technologies and knowledge areas [40]. As smart-environments constitute cyber-physical systems, traditional approaches used in a single discipline, e.g. software engineering, or product engineering, are not sufficient.

Furthermore, effective smart environments need to be considered as sociotechnical systems. As such, besides technological developments, the design of these systems needs to also consider other elements, e.g. people, processes, organizational structures, culture, and surrounding environment.

In terms of system operation, besides the typical maintenance activities involved in traditional product engineering and software engineering, new dimensions have to be considered due to the heterogeneity, diversity, and possible autonomy of components, and interaction between different "communities / ecosystems" (e.g. home environment and external environments). Moreover, smart environments are not designed and built at once rather they gradually grow and/or evolve. For instance, in a smart home environment, new artefacts are incrementally added and/or existing artefacts are from time to time replaced by new ones.

Engineering methods need to be user-centric and also take into account the requirements of scalability and dynamism, multi-stakeholder collaboration and trustworthiness certification, maintenance and evolution, and support for user involvement in co-innovation processes.

\section{$4 \quad$ Concluding Remarks}

The convergence of developments in several basic technologies - e.g. sensors, devices, networking / pervasive computing - enabled good progress on the materialization of the smart environments paradigm, as already illustrated by several application cases.

As new cases emerge and new applications are devised, complexity increases and thus new conceptual, technological, and methodological approaches are needed. In this context, collaborative networks can bring an important contribution to the next generation of smart environments.

Particularly when moving from smart to intelligent environments, increasingly involving components / sub-systems with higher levels of autonomy and decisionmaking capability, looking at these systems from a collaborative networks lens facilitates architectural design and planning governance principles.

Acknowledgments. This work was funded in part by the European Commission through the GloNet project (FP7 programme). 


\section{References}

1. Weiser, M.: The computer of the twenty-first century. Scientific American 265(3), 94-104 (1991)

2. Cook, D.J., Das, S.K.: How smart are our environments? An updated look at the state of the art. Journal Pervasive and Mobile Computing 3(2), 53-73 (2007)

3. Augusto, J.C., Callaghan, V., Cook, D., Kameas, A., Satoh, I.: Intelligent Environments: a manifesto. Human-Centric Computing and Information Sciences 3, 12 (2013)

4. Cook, D.J., Augusto, J.C., Jakkula, V.R.: Ambient intelligence: Technologies, applications, and opportunities. Pervasive and Mobile Computing 5, 277-298 (2009)

5. Acampora, G., Cook, D.J., Rashidi, P., Vasilakos, A.V.: A Survey on Ambient Intelligence in Health Care. Proceedings of the IEEE 101(12), 2470-2494 (2013)

6. Kerner, A., Simunic, D., Prasad, R.: Ambient Intelligence as One of the Key ICT factors for Energy Efficiency in Buildings. Journal of Green Engineering 3, 219-243 (2013)

7. Mckenna, H.P., Arnone, M.P., Chauncey, S.A.: Social Interactions with Wireless Grids: Conceptualizing the 21st Century Ambient Information Soc., pp. 36-41. IEEE Xplore (2013)

8. AAL: Ambient Assisted Living - Catalog of Projects 2013. Ambient Assisted Living joint Programme (2013), http: / /www. aal-europe. eu/wpcontent/uploads/2013/09/AALCatalogue2013_Final.pdf

9. Camarinha-Matos, L.M., Rosas, J., Oliveira, A.I., Ferrada, F.: Care Services Ecosystem for Ambient Assisted Living. J. Enterprise Information Systems (to appear, 2014)

10. Sun, H., De Florio, V., Gui, N., Blondia, C.: Promises and Challenges of Ambient Assisted Living Systems. In: Proc. of the 6th Intern. Conf. on Information Technology: New Generations, pp. 1201-1207. IEEE Xplore (2009)

11. Van Den Broek, G., Cavallo, F., Wehrmann, C.: AALIANCE Ambient Assisted Living Roadmap. IOS Press (2010)

12. Camarinha-Matos, L.M., Afsarmanesh, H.: TeleCARE: Collaborative virtual elderly care support communities. The Journal on Information Technology in Healthcare 2(2), 73-86 (2004)

13. Afsarmanesh, H., Guevara-Masis, V., Hertzberger, L.: Management of Federated Information in Tele-assistance Environments. The Journal on Information Technology in Healthcare 2(2), 87-108 (2004)

14. Rosas, J., Camarinha-Matos, L.M., Carvalho, G., Oliveira, A.I., Ferrada, F.: Development of an Ecosystem for Ambient Assisted Living. In: Rybarczyk, Y., Cardoso, T., Rosas, J., Camarinha-Matos, L.M. (eds.) eNTERFACE 2013. IFIP AICT, vol. 425, pp. 200-227. Springer, Heidelberg (2014)

15. Camarinha-Matos, L.M., Afsarmanesh, H.: Active Aging with Collaborative Networks. IEEE Technology and Society Magazine 30(4), 12-25 (2011)

16. Camarinha-Matos, L.M., Afsarmanesh, H., Ferrada, F., Oliveira, A.I., Rosas, J.: A comprehensive research roadmap for ICT and ageing. Studies in Informatics and Control 22(3), 233-254 (2013)

17. FInES: Embarking on New Orientations Towards Horizon 2020. Position Paper, European Commission (2013), http: / /www. fines-cluster.eu/jm/Documents / Download-document/409-FInES-Horizon-2020_Position-Paper-v2 . 0_final.html 
18. Thoma, M., Antonescu, A.-F., Mintsi, T., Braun, T.: Linked Services for Enabling Interoperability in the Sensing Enterprise. In: van Sinderen, M., Oude Luttighuis, P., Folmer, E., Bosems, S. (eds.) IWEI 2013. LNBIP, vol. 144, pp. 131-144. Springer, Heidelberg (2013)

19. NFS: Cyber-Physical Systems (CPS) (2012), http://www.nsf.gov/funding/ pgm_summ.jsp?pims_id=503286 (accessed on June 17, 2014)

20. Sundmaeker, H., Guillemin, P., Friess, P., Woelfflé, S. (eds.): Vision and Challenges for Realising the Internet of Things. CERP-IoT, European Commission (2010)

21. Camarinha-Matos, L.M., Goes, J., Gomes, L., Martins, J.: Contributing to the Internet of Things. In: Camarinha-Matos, L.M., Tomic, S., Graça, P. (eds.) DoCEIS 2013. IFIP AICT, vol. 394, pp. 3-12. Springer, Heidelberg (2013)

22. Jeschke, S.: Everything 4.0? - Drivers and Challenges of Cyber Physical Systems. RWTH University Aachen (2013)

23. Li, W., Bao, J., Shen, W.: Collaborative wireless sensor networks: A survey. In: SMC 2011 IEEE International Conference on Systems, Man, and Cybernetics, Anchorage, AK, USA, October 9-12, pp. 2614-2619. IEEE Xplore (2011)

24. Allameh, E., Heidari Jozam, M., de Vries, B., Timmermans, H.J.P., Beetz, J.: Smart Home as a smart real estate: a state of the art review. In: 18th International Conference of European Real Estate Society, ERES 2011, Eindhoven, Netherlands (2011)

25. Alam, M.R., Reaz, M.B.I., Ali, M.A.M.: A Review of Smart Homes-Past, Present, and Future. IEEE Trans. Systems, Man and Cybernetics C 42(6), 1190-1203 (2013)

26. OPENHAB: openHAB Open Source Automation Server (2014), http://www . openhab.org

27. FHEM: FHEM Home Automation Sever (2014), http : / / www . fhem. de

28. OPENREMOTE: openRemote Open Source Automation Platform (2014), http: / / www. openremote.org

29. Gharavi, H., Ghafurian, R.: Smart Grid: The Electric Energy System of the Future. Proceedings of the IEEE 99(6), 917-921 (2011)

30. Neßelrath, R., Lu, C., Schulz, C.H., Frey, J., Alexandersson, J.: A Gesture Based System for Context - Sensitive Interaction with Smart Homes. In: Wichert, R., Eberhardt, B. (eds.) Ambient Assisted Living, vol. 63, pp. 209-219. Springer, Heidelberg (2011)

31. Borja, R., de la Pinta, J.R., Álvarez, A., Maestre, J.M.: Integration of service robots in the smart home by means of UPnP: A surveillance robot case study. Robotics and Autonomous Systems 61(2), 153-160 (2013)

32. Neirotti, P., Marco, A., Cagliano, A.C., Mangano, G., Scorrano, F.: Current trends in Smart City initiatives: Some stylised facts. Cities 38, 25-36 (2014)

33. Giffinger, R., Gudrun, H.: Smart cities ranking: an effective instrument for the positioning of the cities? ACE: Architecture, City and Environment 4(12), 7-26 (2010)

34. García-Murillo, M., Vélez Ospina, J.A.: The paradox of intelligent infrastructures. Redes.com, $\mathrm{n}^{\mathbf{0}}$ 9, pp. 183-209 (2014), http://revista-redes.hospeda gemdesites.ws/index.php/revista-redes/article/view/302/343

35. Osório, A.L., Camarinha-Matos, L.M., Gomes, J.S.: A collaborative case study: The extended "ViaVerde" toll payment system. In: Collaborative Networks and their Breeding Environments, pp. 559-568. Springer

36. Weber, K.M., Heller-Schuh, B., Godoe, H., Roeste, R.: ICT-enabled system innovations in public services: Experiences from intelligent transport systems. Telecommunications Policy 38(5-6), 539-557 (2014) 
37. Fon, Z., Kulkarni, P., Gormus, S., Efthymiou, C., Kalogridis, G., Sooriyabandara, M., Zhu, Z., Lambotharan, S., Chin, W.H.: Smart Grid Communications: Overview of Research Challenges, Solutions, and Standardization Activities. IEEE Communications Surveys \& Tutorials 15(1), 21-38 (2013)

38. Farhangi, H.: A Road Map to Integration: Perspectives on Smart Grid Development. IEEE Power and Energy Magazine 12(3), 52-66 (2013)

39. Camarinha-Matos, L.M., Afsarmanesh, H., Boucher, X.: The Role of Collaborative Networks in Sustainability. In: Camarinha-Matos, L.M., Boucher, X., Afsarmanesh, H. (eds.) PRO-VE 2010. IFIP AICT, vol. 336, pp. 1-16. Springer, Heidelberg (2010)

40. Broy, M., Schmidt, A.: Challenges in Engineering Cyber-Physical Systems. Computer (February 2014)

41. Ortner, E., Schneider, T.: Temporal and Modal Logic Based Event Languages for the Development of Reactive Application Systems. In: Proc. 1st Int. Workshop on Complex Event Processing for the Future Internet, Vienna, Austria, September 28-30 (2008)

42. Franco, R., Ortiz Bas, Á., Lario Esteban, F.: Modeling extended manufacturing processes with service-oriented entities. Service Business 3(1), 31-50 (2009)

43. Cardoso, T., Camarinha-Matos, L.M.: Pro-Active Service Ecosystem Framework. To appear in International Journal of Computer Integrated Manufacturing 26(11), 1021-1041 (2013) 